\title{
BERICHT ÜBER DIE UMSTELLUNG DES ANGEBOTES DER TEACHING LIBRARY AN DER UNIVERSITÄTSBIBLIOTHEK DER MEDIZINISCHEN UNIVERSITÄT WIEN AUF DIGITALE FORMATE IM ZUGE DER COVID-19-PANDEMIE 2020
}

\author{
von Lukas Zach und Helmut Dollfuß
}

Zusammenfassung: In dem Beitrag wird über die Umstellung des Lehrangebots der Teaching Library an der Universitätsbibliothek der Medizinischen Universität Wien berichtet. Die aktuelle COVID-19-Situation und die damit einhergehenden Zugangsbeschränkungen seitens des AKH Wien machten es notwendig, das gesamte Angebot der Teaching Library auf digitale Formate umzustellen. Davon betroffen waren klassische Bibliotheksführungen genauso wie mehrere Lehrveranstaltungen, die im Rahmen der Curricula der Medizinischen Universität Wien und der Karl Landsteiner Privatuniversität für Gesundheitswissenschaften abgehalten wurden. Die Situation machte eine umfangreiche Neukonzeption der Inhalte sowie interne technische Schulungen notwendig. Es hat sich gezeigt, dass das digitale Angebot von den Studierenden gut angenommen wurde. Jedoch machte das neue Format einen frontaleren Unterricht notwendig, da Übungen im herkömmlichen Sinne kaum umsetzbar waren.

Schlagwörter: Medizinische Universität Wien; COVID-19; Teaching Library; Fernunterricht; Online-Unterricht; Webex; Moodle; Bericht

\section{REPORT ON HOW THE TEACHING LIBRARY OF THE UNIVERSITY LIBRARY OF THE MEDICAL UNIVERSITY OF VIENNA CHANGED ITS COURSE OFFER TO DIGITAL FORMATS DUE TO THE COVID-19 PANDEMIC IN 2020}

\begin{abstract}
The article describes the restructuring of the courses and lectures held by the teaching library of the University Library of the Medical University of Vienna. The current restriction of access to the General Hospital of Vienna (AKH Wien) due to the ongoing COVID-19 pandemic required the remodelling of the services offered into digital formats. This included library introductions as well as lectures that were part of the curricula of both the Medical University of Vienna and the Karl Landsteiner University for Health Sciences. The situation called for in-house software training sessions and an extensive re-conception of the content of the courses. The new online courses were well received by the students. It has become apparent, however, that a more frontal approach to teaching was necessary since conventional tutorials could not be held online.
\end{abstract}


Keywords: Medical University of Vienna; COVID-19; teaching library; distant teaching; online-teaching; Webex; Moodle; report

DOI: https://doi.org/10.31263/voebm.v73i3-4.5342

() Lukas Zach, Helmut Dollfuß

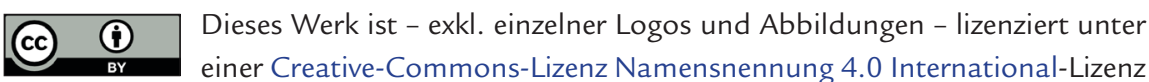

\section{Einleitung}

Die weltweite COVID-19-Pandemie hat im Laufe des Jahres so gut wie jeden Aspekt des privaten und öffentlichen Lebens getroffen. So waren und sind auch Bibliotheken gefordert, ihre Angebote den sich ständig ändernden Rahmenbedingungen anzupassen. Diese Entwicklung machte auch vor dem Team Teaching Library an der Universitätsbibliothek der Medizinischen Universität Wien (UB MedUni Wien) nicht Halt und machte es notwendig, das Angebot grundlegend umzugestalten und zum Teil auf völlig neue Beine zu stellen.

\section{Angebote der Teaching Library der UB MedUni Wien}

Das Team Teaching Library an der UB der MedUni Wien besteht aus sieben Personen, die jedoch größtenteils in anderen Abteilungen ihrer Haupttätigkeit nachgehen. Das Angebot umfasst ein breit gefächertes Spektrum an Schulungen, Kursen, Workshops und Führungen zu allerlei Themen aus dem bibliothekarischen Kontext. Über das Jahr 2019 etwa wurden an der UB MedUni Wien 36 Bibliotheksführungen durchgeführt. Davon richteten sich 30 an Pflegeschüler*innen und 6 an Schüler*innen, die gerade ihre vorwissenschaftliche Arbeit (VWA) schreiben. In der zweiten Septemberhälfte, kurz vor Semesterbeginn, bietet die UB auch immer Kurzführungen für Erstsemestrige durch die Bibliothek an. Diese 15-20-minütigen Führungen finden im Rahmen einer Campustour statt, die jedes Jahr von der $\mathrm{ÖH}-\mathrm{Med}$ an der Medizinischen Universität organisiert wird und die von den Studierenden sehr gut angenommen wird. Innerhalb von zwei Wochen besuchen im Zuge dessen ungefähr 30 Kleingruppen die Bibliothek - das entspricht 3-4 Führungen pro Tag. Insgesamt kommen so etwa 450 erstsemestrige Studierende jedes Jahr in die Bibliothek. Abgesehen von Biblio- 
theksführungen bietet das Team Teaching Library auch MedUni-interne Seminare zur Personalentwicklung an, in denen es vor allem um die Nutzung der elektronischen Informationsquellen geht.

Eine Besonderheit im Angebot der Teaching Library an der Medizinischen Universität Wien ist die Tatsache, dass die Bibliothek mit mittlerweile zwei Pflichtlehrveranstaltungen am Curriculum beteiligt ist. Bereits seit 2001 ist die Bibliothek mit einer Übung zum Thema „Wege zum Volltext“ im 3. Semester vertreten (Dollfuß und Miehl 2019). Bei dieser 45-minütigen Veranstaltung mit Anwesenheitspflicht werden verschiedene Wege von einem Literaturzitat zum Volltext aufgezeigt, wobei ein starker Fokus auf der aktiven Mitarbeit der Studierenden in Form von zahlreichen Übungen liegt. Hier nehmen jedes Jahr rund 750 Studierende teil.

Seit jeher wurde diese Übung in Kleingruppen zu je ca. 30 Studierenden in dem PC-Raum der Bibliothek durchgeführt, in dem 16 PCs zur Verfügung stehen. Eben diese beengte Raumsituation machte es früh klar, dass diese Übungen im Wintersemester 2020/21 wohl nicht im gewohnten Rahmen stattfinden würden können. Dies betraf auch Lehrveranstaltungen, die im Rahmen des Curriculums für Humanmedizin der Karl Landsteiner Privatuniversität für Gesundheitswissenschaften (KLU) in Krems vom Team Teaching Library im selben PC-Raum gehalten wurden.

Zusätzlich zu diesen beiden, schon länger durchgeführten Übungen, kam im Wintersemester 2020/21 eine weitere Lehrveranstaltung dazu, in der eine Einführung in die Bibliothek sowie ein Überblick über das medizinische Publikationswesen und über die Angebote der UB präsentiert werden. Diese Kleingruppenübung findet im 1. Semester statt und unterscheidet sich inhaltlich stark von jener im 3. Semester. An dieser Lehrveranstaltung nehmen insgesamt ca. 750 Studierende teil. Diese Übung wurde komplett neu erarbeitet und direkt als Onlineübung konzipiert, da es hier keine Erfahrung im Präsenzunterricht aus den vergangenen Jahren gab.

\section{Auswirkungen der COVID-19-Maßnahmen seitens des AKH Wien}

Das AKH Wien, in dem sich auch die Räumlichkeiten der UB MedUni Wien befinden, reagierte schnell auf die sich abzeichnende Pandemie und verhängte bereits ab 15. März 2020 strenge Zugangskontrollen für das Gebäude. Der Zutritt zum AKH ist seitdem nur für Mitarbeiter*innen sowie Patient* innen mit einem Termin bzw. einem dringenden Notfall gestattet. So stand schnell fest, dass sowohl Studierende als auch externe Benutzer*innen bis auf weiteres keinen Zugang zur Bibliothek mehr haben würden. 
Als erste spürbare Auswirkung wurden sukzessive alle für 2020 bereits ausgemachten Termine für externe Führungen storniert. Dies betraf 13 Führungen für Pflegeschüler*innen sowie eine WWA-Führung.

\section{Umstellung auf Distant Teaching}

Die Medizinische Universität Wien hat Mitte März als Reaktion auf den Lockdown die Telekonferenz-Software Webex campusweit lizenziert und deren Nutzung für sämtliche Besprechungen und Lehrveranstaltungen forciert (Dollfuß 2020). Dies kam uns Bibliothekar*innen entgegen, da bereits während des Umstiegs auf Alma diese Software für viele Besprechungen und Jours fixes genutzt wurde und daher bereits eine gewisse Erfahrung vorhanden war. Webex bietet unterschiedliche Module, um von einer einfachen Besprechung bis hin zu einer Vorlesung mit hunderten Zuhörer*innen sämtliche Szenarien abdecken zu können.

Neben Webex für die Abhaltung der Übungen wird an der Medizinischen Universität Wien auch die Lernplattform Moodle verwendet. Dieses Programm bietet Vortragenden sehr viele Möglichkeiten, um Lehrveranstaltungen online zu begleiten. Von vorbereitender Lektüre über Skripten bis hin zu kleinen Übungen oder Videoaufzeichnungen steht ein großes Repertoire an Funktionen zur Verfügung.

Für beide Programme wurden seitens der Universität umfangreiche Schulungs- und Fortbildungsangebote über die Personalentwicklung angeboten, die von den Mitgliedern des Teams Teaching Library rege genutzt wurden. Vor allem die Schulungen zur Erstellung von Kursen in Moodle waren sehr aufschlussreich und hatten zur Folge, dass es erstmals sehr detailliert ausgearbeitete Online-Kurse für die beiden seitens der Bibliothek durchgeführten Lehrveranstaltungen gab.

Da die Kurse der UB erst im Wintersemester stattfanden, blieb zum Glück genügend Zeit für eine gründliche Vorbereitung und eine umfangreiche Neukonzipierung des Angebotes.

Bei zwei der drei vorzubereitenden Lehrveranstaltungen gab es bereits aus den vergangenen Jahren etablierte inhaltliche Konzepte sowie entsprechende Erfahrungswerte auf die man aufbauen konnte. Die erste Idee, den Aufbau der letzten Jahre eins zu eins zu übernehmen, musste jedoch recht schnell wieder fallen gelassen werden. Normalerweise handelt es sich um Kleingruppenübungen mit einem sehr starken Fokus auf die Mitarbeit der Studierenden. Der bisherige didaktische Aufbau sah nur kurze Blöcke von theoretischem Vortrag vor, während der Großteil der akademischen Einheit 
(45 Minuten) für praktische Übungen am PC und deren intensive Betreuung reserviert war. Ein wesentlicher Teil des Lehrinhaltes wurde von den Studierenden im Laufe dieser Übungsblöcke selbst erarbeitet. Diese Vorgehensweise ist bei einer Online-Übung jedoch nicht umsetzbar, da es unmöglich wäre, die Ergebnisse sämtlicher Studierenden in Echtzeit zu sichten und individuell zu korrigieren. Die Gefahr, dass sich hier Fehler einschleichen und dann aufgrund mangelnder Korrektur als richtig eingestuft werden, ist zu groß. Erschwerend kam hinzu, dass die Übung im 3. Semester seitens der Curriculumsleitung von Kleingruppen auf Webex-Großgruppen umgestellt wurde. Dadurch veränderte sich das ganze Wesen der Lehrveranstaltung: statt mehrerer Kleingruppen zu je 30 Personen waren es dieses Semester nur mehr drei Gruppen, mit jeweils 250 Studierenden. Aufgrund dieser Tatsache, und der kaum vorhandenen Durchführbarkeit von Übungen, wurde entschieden, die Lehrveranstaltung als Frontalunterricht zu gestalten. Die Inhalte wurden entsprechend adaptiert, so wurden die theoretischen Teile vertieft und ausgebaut, während die ursprünglich zahlreichen Übungen auf einige wenige Beispiele reduziert wurden. Diese Beispiele wurden über den geteilten Bildschirm der Vortragenden vorgeführt und dann gemeinsam besprochen. Um den eigentlichen Übungscharakter der Lehrveranstaltung nicht gänzlich zu vernachlässigen, wurde versucht, über die „Feedback“-Funktion in Webex die Studierenden zu aktivieren. Hier bietet das Programm die Möglichkeit, per Knopfdruck Feedback wie etwa „Ja“, „Nein“, „zu schnell“, „bitte lauter“, etc. zu geben. Da diese Variante jedoch nur schlichte Ja-Nein-Fragen ermöglichte, war die praktische Implementierung auf Verständnisfragen und Einschätzungen („Nach dem, was Sie gehört haben, glauben Sie, dass...?") limitiert.

Ergänzend zum Vortrag über Webex wurde ein umfangreicher Online-Kurs auf der Lernplattform Moodle eingerichtet. Da der Vortrag selbst zum größten Teil aus Live-Präsentationen im Internet bestand, gab es keine PowerPoint-Präsentation, die man hätte hochladen können. Aus diesem Grund wurde der gesamte theoretische Inhalt aufwändig mit zahlreichen eigens dafür verfassten Texten, Screenshots sowie Kurzvideos aufbereitet und auf Moodle gestellt. Um den Übungscharakter zu betonen, wurden zusätzlich kleine Übungen und Wissensüberprüfungen in Form von Multiple-Choice-Fragen eingebaut. Diese Tests waren auf freiwilliger Basis und auch wiederholbar. Da Moodle automatisch jegliche Aktivität dokumentiert, hatte diese Vorgangsweise den praktischen Nebeneffekt, dass auch gleich eine Ersatzleistung parat war. Hatte ein Student* eine Studentin den Webex-Kurs verpasst, genügte es, den Moodle-Kurs durchzuarbeiten und die Fragen zu beantworten. 
Die Lehrveranstaltung für die Studierenden im dritten Semester wurde als erste erarbeitet, da sie gleich zu Beginn des Wintersemesters 2020/21 stattfand. Die im Zuge dieser Neukonzeption gewonnenen Erfahrungen flossen dann in die Gestaltung der beiden anderen Übungen ein: jene(r) für die Erstsemestrigen und jene(r) für die Studierenden der KLU. Auch hier zeigte sich, dass der eigentlich geplante Übungscharakter durch einen frontaleren Unterricht ersetzt werden musste. Diese beiden Lehrveranstaltungen wurden jedoch in Kleingruppen zu je 25-30 Personen abgehalten, wodurch eine stärkere Einbindung der Studierenden durch die vermehrte Verwendung der Feedback-Funktionen möglich war.

Sämtliche Lehrveranstaltungen wurden immer von zwei Angehörigen aus dem Team Teaching Library betreut. Während eine Person den Bildschirm teilte und die Übung leitete, überwachte die andere Person den Chatverlauf sowie die Feedbacks. Diese Aufteilung erwies sich in der Praxis als sehr hilfreich, da die Chatfunktion mitunter sehr rege genutzt wurde, sodass in manchen Gruppen alle paar Minuten eine Frage gestellt wurde.

Während die Lehrveranstaltungen aus den Curricula auf diese Weise stattfinden konnten, wurden keine herkömmlichen Bibliotheksführungen seit März 2020 durchgeführt. Solche Führungen werden vor allem von Schüler*innen von Krankenpflegeschulen oder medizinisch-technischen Ausbildungszweigen in Anspruch genommen. Vereinzelt nutzen auch WWA-Klassen dieses Angebot. Das Programm dieser Führungen wird stets auf die jeweiligen Bedürfnisse der Gruppe abgestimmt, beinhaltet aber meistens eine Führung durch die Räumlichkeiten der Bibliothek sowie eine anschließende Katalog- und Rechercheschulung im PC-Raum der UB MedUni Wien. Nachdem sämtliche bereits gebuchten Führungen im Laufe des März und April 2020 storniert wurden, wurde den Lehrer*innen der betroffenen Gruppen die Möglichkeit einer Ersatzveranstaltung in Form eines Online-Seminars via Webex vorgeschlagen. Der ausgefallene Führungsteil durch den Freihandbereich hätte durch Fotos und einen Lageplan ersetzt werden können und die Rechercheschulungen hätten über den geteilten Bildschirm gezeigt werden können. Diese Angebote wurden jedoch seitens der Lehrer*innen nicht angenommen, sodass hier auch keine weiteren Konzepte erarbeitet wurden.

\section{Fazit}

Rückblickend hat der Umstieg auf Distant Teaching seitens des Teams Teaching Library der UB MedUni Wien sehr gut funktioniert, was nicht zuletzt auch an der langen Vorlaufzeit von rund sechs Monaten lag. Ein weiterer 
Vorteil war sicherlich, dass Teile des Teams die zu verwendende Software bereits aus der Vergangenheit kannten und im Umgang damit geschult waren. Nichtsdestotrotz waren die seitens der MedUni Wien angebotenen Trainings zu Webex und Moodle sehr hilfreich. Hätte man sich dieses Wissen selbst erarbeiten müssen, hätte das sicherlich sehr viel mehr Zeit in Anspruch genommen.

Was den Inhalt der Lehrveranstaltungen anbelangt, war es enttäuschend, den Übungsaspekt hinten anstellen zu müssen. Ein beinahe ausschließlicher Frontalunterricht ist für diese Art der praxisbezogenen Inhalte nicht die optimale didaktische Methode. Dieser Umstand wurde den Studierenden offen kommuniziert und stieß auch auf viel Verständnis.

Das Feedback der Studierenden - sowohl während der Übungen, als auch später in der Evaluation - war dennoch sehr positiv. Dies hat sicher auch damit zu tun, dass hier schon eine gewisse Erfahrung im Distant Learning vorhanden war, bzw. dass die Erstsemestrigen noch nichts Anderes kannten.

Innerhalb des Teams Teaching Library wurde vor allem der fehlende visuelle Kontakt mit den Studierenden als unangenehm empfunden. Es macht einen riesigen Unterschied, ob man in einem Seminarraum 30 Personen gegenübersteht oder ob man eine Stunde lang mit dem Monitor spricht. So war es auch schwer einzuschätzen, ob der gebrachte Inhalt den Erwartungen und dem Niveau der Studierenden entsprach. Körpersprache ist in der Lehre enorm wichtig und sehr aussagekräftig!

An diverse technische Probleme hat man sich mittlerweile in Zeiten der Telekonferenzen und des Homeoffice bereits gewöhnt und auch diese Lehrveranstaltungen blieben davon nicht verschont. Schlechte Internetverbindung, Bild- und Tonprobleme, Verbindungsverluste, Personen, die nicht in das Webex Event kommen, etc. kamen zwar vor, doch war die Häufigkeit überschaubar. Auch konnten diese Probleme alle letztendlich gelöst werden. Hier sollte man ein gewisses Maß an Geduld aufbringen und sich vor allem während des Vortrags nicht aus der Ruhe bringen lassen. Wie oben bereits erwähnt, hat sich die Entscheidung, immer zu zweit eine Übung zu betreuen, als sehr wichtig herausgestellt. Natürlich kann man auch alleine eine Lehrveranstaltung via Distant Teaching durchführen, doch sollte man in diesem Fall schon über eine gewisse Erfahrung und Sicherheit mit der verwendeten Software verfügen.

Die eigens zur Begleitung der Webex-Seminare erarbeiteten MoodleKurse stellen eine große Bereicherung für das Angebot des Teams Teaching Library dar und werden daher auch in Zukunft in der Lehre eingesetzt werden. Hat man sich einmal die Mühe gemacht, solche Kurse detailliert 
auszuarbeiten, genügt es meist, sie in den kommenden Jahren lediglich zu aktualisieren und die Inhalte auf den neuesten Stand zu bringen. Ohne den Lockdown und die Umstellung auf Distant Teaching würden diese überaus praktischen und gut genutzten Kurse wohl nicht existieren.

Mag. Lukas Zach

ORCID iD: https://orcid.org/0000-0001-8949-6900 Medizinische Universität Wien, Universitätsbibliothek

E-Mail: lukas.zach@meduniwien.ac.at

Ing. Mag. Helmut Dollfuß, MSc

ORCID iD: https://orcid.org/0000-0001-5106-5945 Medizinische Universität Wien, Universitätsbibliothek

E-Mail: helmut.dollfuss@meduniwien.ac.at

\section{Literatur}

Dollfuß, H.; Miehl, M. (2019). Wege zum elektronischen Volltext. In: Horn, W. (Hrsg.): Wissenschaft und Medizin: ein Lehrbuch für das erste Spezielle Studienmodul (SSM 1) - Block 7. 16. Aufl. Facultas: Wien.

Dollfuß, H. (2020). Virtuelles Treffen der VÖB-Kommission Informationskompetenz". Mitteilungen der Vereinigung Österreichischer Bibliothekarinnen und Bibliothekare, 73(2), S. 203-206. https://doi.org/10.31263/ voebm.v73i2.3982 